\title{
Improved concentration and separation of particles in a 3D dielectrophoretic chip integrating focusing, aligning and trapping
}

\author{
Ming Li $\cdot$ Shunbo Li $\cdot$ Wenbin Cao $\cdot$ \\ Weihua Li · Weijia Wen · Gursel Alici
}

Received: 20 July 2012/ Accepted: 26 September 2012

(C) Springer-Verlag Berlin Heidelberg 2012

\begin{abstract}
This article presents a dielectrophoresis (DEP)based microfluidic device with the three-dimensional (3D) microelectrode configuration for concentrating and separating particles in a continuous throughflow. The $3 \mathrm{D}$ electrode structure, where microelectrode array are patterned on both the top and bottom surfaces of the microchannel, is composed of three units: focusing, aligning and trapping. As particles flowing through the microfluidic channel, they are firstly focused and aligned by the funnel-shaped and parallel electrode array, respectively, before being captured at the trapping unit due to negative DEP force. For a mixture of two particle populations of different sizes or dielectric properties, with a careful selection of suspending medium and applied field, the population exhibits stronger negative DEP manipulated by the microelectrode array and, therefore, separated from the other population which is easily carried away toward the outlet due to hydrodynamic force. The functionality of the proposed microdevice was verified by concentrating different-sized polystyrene (PS) microparticles and yeast cells dynamically flowing in the microchannel. Moreover, separation based on size and
\end{abstract}

\section{Li · W. Li $(\square) \cdot$ G. Alici}

School of Mechanical, Materials and Mechatronic Engineering, University of Wollongong, Wollongong, NSW 2522, Australia e-mail: weihuali@uow.edu.au

\section{S. Li · W. Wen $(\square)$}

Department of Physics, The Hong Kong University of Science and Technology, Clear Water Bay, Kowloon, Hong Kong e-mail: phwen@ust.hk

\section{W. Cao $\cdot$ W. Wen}

Nano Science and Technology Program

and KAUST-HKUST Micro/Nanofluidic Joint Laboratory,

The Hong Kong University of Science and Technology,

Clear Water Bay, Kowloon, Hong Kong dielectric properties was achieved by sorting PS microparticles, and isolating $5 \mu \mathrm{m}$ PS particles from yeast cells, respectively. The performance of the proposed microconcentrator and separator was also studied, including the threshold voltage at which particles begin to be trapped, variation of cell-trapping efficiency with respect to the applied voltage and flow rate, and the efficiency of separation experiments. The proposed microdevice has various advantages, including multi-functionality, improved manipulation efficiency and throughput, easy fabrication and operation, etc., which shows a great potential for biological, chemical and medical applications.

Keywords 3D electrodes - Dielectrophoresis . Particle concentration · Particle separation - Microfluidics

\section{Introduction}

The development of microdevice for effective bioparticle manipulation, including focusing, concentrating, sorting and patterning, etc. has received an increased attention due to its wide applications in the field of biotechnology, medicine and environment. Different technologies have been employed by various microdevices to manipulate particles in solution, such as mechanical, thermal, magnetic, acoustic, optical, chemical and electrical (electrophoretic and dielectrophoretic) methodologies, which have been reviewed in the relevant literature (Gossett et al. 2010; Roda et al. 2009; Kang and Li 2009). Among these existing technologies, dielectrophoresis (DEP) may be the most popular one, because it offers a more selective, accurate and controllable manipulation of microparticles (Zhang et al. 2010; Wlodkowic and Cooper 2010). Unlike other techniques, DEP depends on the dielectric properties, 
which represent structural, morphological and chemical characteristics of bioparticles, leading to the analysis of high selectivity and sensitivity (Pysher and Hayes 2007). DEP manipulation is also straightforward and fully controllable by varying the electric conductivity of the suspending medium or the frequency and magnitude of the applied electric field. In addition, DEP enables contact-free manipulation of particles with lower sample consumption and fast speed.

DEP, first adopted by Pohl (1978), occurs due to a translational force exerted on a polarizable particle when it is subjected to a nonuniform electric field. The required inhomogeneous electric field for DEP can be obtained by insulating obstacles placed in the microchannel or channel geometry modification (Srivastava et al. 2011), while electrodes are placed at inlet and outlet reservoirs. However, this method suffers from low particle throughput due to the limited electrokinetic flow rate, and is sensitive to cell damage and contamination due to particle adhesion on channel walls (Xuan et al. 2010). These drawbacks can be overcome by arranging microelectrode structures inside the microfluidic channel for electric field nonuniformities. A variety of $2 \mathrm{D}$ or planar microelectrodes normally patterned on the bottom surface of microchannel have been developed, including parallel or interdigitated, castellated, oblique, curved, quadruple, and matrix ones. Different designs of microelectrodes used for DEP have been reviewed by researchers (Zhang et al. 2010; Khoshmanesh et al. 2010; Pethig 2010; Song et al. 2008). Although 2D microelectrodes have been successfully equipped in various DEPbased microdevices for manipulating bioparticles (i.e. DNA, protein, bacteria, virus, mammalian and yeast cells) (Pethig 2010), the use of such configuration can be problematic for some practical applications. This is because DEP force will decrease quickly as the distance from the planar electrodes increase, so that only the motion of a few particles close to the electrode surface can be directed, which is not sufficient especially for trapping and concentration of relatively large numbers of particles. Moreover, interaction with walls or absorption of particles on electrode surface is always an issue (Cetin and Li 2011). As an alternative, 3D electrode structures are built on both the top and bottom surfaces of the channel, where effective DEP forces are created over a larger volume of the microchannel, and the particles are focused and located around the channel center region in the vertical direction. Therefore, the aforementioned problems of 2D planer microelectrodes, such as insufficient holding force, relatively low trapping/ sorting efficiency, and particle adhesion, are avoided.

Microdevices with pairs of oblique electrode arrays on both top and bottom surfaces of the microfluidic channel have been demonstrated by researchers for the accumulation and separation of microparticles (Schnell et al. 1999; Dürr et al. 2003; Chen and Du 2007). However, such systems increase the need of labor and equipment, as different arrays of microelectrodes were excited independently, moreover, particles are directed to the accumulation area placing at the corner formed by the channel wall and microelectrode, leading to high risk of particle-wall interaction and particle absorption to channel walls. Yasukawa et al. (2007) used microelectrode arrays composing of navigator, cap and collector electrodes on two substrates in a microfluidic system for immunoassays. Polystyrene microparticles modified with anti-mouse IgG were manipulated and captured in the caged area. But the particle separating experiments were not tried and the capturing performance of microsystem was not discussed. Cheng et al. (2007) developed an integrated 3D DEP-based chip for continuous manipulation of bioparticles, but trapped live cells on the filter electrode, due to positive DEP, are not easily released and are at high risk of biological damage arising from highstrength electric field. Furthermore, multiple power supplies are also required to excite discrete microelectrode arrays. 3D focusing of particles has been achieved by patterning microelectrodes on the surface of an elliptic-like microchannel (Yu et al. 2005) and combining X-patterned insulating microstructures and bi-layer microelectrode (Jen et al. 2011). However, the fabrication process, including etching of glass wafers, bonding based on heat, pressure and adhesion is relatively expensive, time-consuming, and requires aggressive chemicals. In addition, 3D microelectrode configurations formed by building extruded electrodes on the bottom substrate (Voldman et al. 2003; Iliescu et al. 2008, 2009; Tai et al. 2007) and electrode patterns on the surface of sidewalls (Cetin et al. 2009; Lewpiriyawong et al. 2010, 2011; Wang et al. 2007, 2009) have been employed in microdevices for particle manipulation. However, the fabrication techniques are more complicated.

In this work, we present a microfluidic device equipped with 3D microelectrode arrays patterned on both the bottom and top surfaces of microchannel for particle concentration and separation using negative DEP. The 3D electrode structure is composed of a funnel-shaped focusing unit, a parallel aligning unit and a crescent-shaped trapping unit in series, which enables integrated functionality as concentration of particles in a continuous flow and separation of particle mixture according to size and dielectric properties. Numerical simulations were performed to optimize the electrode design. The focus-aligntrap functioning of the microdevice was demonstrated using PS particles with 5,10 and $15 \mu \mathrm{m}$ in diameter and yeast cells. Moreover, the threshold voltages required for successfully trapping varying-sized particles were presented, along with the effects of applied voltage and flow rate on the cell-trapping efficiency. The microdevice serving as a microseparator was verified by separating 
microparticles according to size and separating yeast cells from $5 \mu \mathrm{m}$ diameter PS particles according to dielectric properties. Evaluation of the separating efficiency was also carried out by counting and comparing the number of each particle population at the inlet and outlet.

\section{Theory}

The time-averaged DEP force acting on a spherical particle is given by Jones (2003)

$$
F_{\mathrm{DEP}}=2 \pi r^{3} \varepsilon_{\mathrm{m}} \operatorname{Re}\left[f_{\mathrm{CM}}\right] \nabla E^{2}
$$

where $r$ is the radius of the particle, $\varepsilon_{\mathrm{m}}$ is the permittivity of the suspending medium, $E$ is the root-mean-square (rms) value of the electric field, and $\operatorname{Re}\left[f_{\mathrm{CM}}\right]$ is the real part of the Clausius-Mossotti (CM) factor, represented as

$f_{\mathrm{CM}}=\frac{\varepsilon_{\mathrm{p}}^{*}-\varepsilon_{\mathrm{m}}^{*}}{\varepsilon_{\mathrm{p}}^{*}+2 \varepsilon_{\mathrm{m}}^{*}}$

where $\varepsilon_{\mathrm{p}}^{*}$ and $\varepsilon_{\mathrm{m}}^{*}$ are the complex permittivities of the particle and suspending medium, respectively, each can be written as

$\varepsilon^{*}=\varepsilon-i \sigma / \omega$

where $i=\sqrt{-1}, \sigma$ is the electric conductivity and $\omega$ is angular frequency of the applied electric field. The DEP force is dependent on the dielectric properties of the particle and the suspending medium, particle size and frequency of applied electric field. Based on the polarity of the $\operatorname{Re}\left[f_{\mathrm{CM}}\right]$, the DEP force can be positive $\left(\operatorname{Re}\left[f_{\mathrm{CM}}\right]>0\right)$, which attracts particles toward the regions of high electric field, and negative $\left(\operatorname{Re}\left[f_{\mathrm{CM}}\right]<0\right)$, which repels particles from the regions of high electric field.

The hydrodynamic force applied on a particle can be defined as

$F_{\mathrm{HD}}=6 \pi \eta r v$

where $\eta$ and $v$ are the viscosity and velocity of the fluid, respectively. It can be found that the hydrodynamic force is linearly proportional to the fluid velocity and particle size. The movement of the particle is determined by the combined effect of both forces: DEP force and hydrodynamic force. In order to direct and trap the particles, the DEP force in the direction of flow must be stronger than the hydrodynamic force.

\section{Numerical simulation}

The key point in achieving desirable DEP effect is the generation of electric field gradient by the electrode array. Numerical simulations were performed using commercial software COMSOL 4.0 (COMSOL Inc., Burlington, MA). For simplicity, a 2D model was employed without considering the effect of channel wall on electric field distribution. Figure 1 depicted the electric field distribution (contours) and direction of negative DEP force (black arrows) in a vertical plane of $30 \mu \mathrm{m}$ height microchannel, when a voltage of $5 \mathrm{~V}$ is applied to the microelectrodes patterned on top and bottom surfaces. The numerical results indicated that non-uniform electric field symmetric about the channel centerline was generated, with the maximum strength obtained at the electrode edge, and electric field of high strength covers almost the whole height of the microchannel. Particles can be directed toward the center of microchannel under the effect of negative DEP force pointing from the electrode region to the channel center region. Therefore, 3D electrode configuration addresses the problems encountered by 2D planer electrodes, such as insufficient DEP force and particle levitation.

In order to optimize the design of electrode structure, the distribution of the electric field and electric potential in three types of electrode geometry were compared: (1) triangular, (2) rectangular, and (3) crescent shape. Both the width of the microelectrodes and the electrode spacing at the aperture were set at the same values of 40 and $80 \mu \mathrm{m}$, respectively. Figure 2 a shows the contours of electric field $(E)$ within the region formed by different electrode shapes at an applied voltage of $5 \mathrm{~V}$. The electric potential applied to the microelectrodes generates nonuniform electric field in all the cases. It is known that the electric field is constricted at the corner, thus, a stronger and highly non-uniform electric field is created at the sharp corners (dark blue region corresponds to strong electric field). Due to the curved shape, the crescent-shaped microelectrode created a smoothly varied electric field within the microchannel, eliminating locally amplified electric field due to sharp corners. Compared to triangular (a1) and rectangular (a2) microelectrodes, where electric fields are locally concentrated at the sharp corners (marked by red dashed circles), the electric field is generated more uniformly within the region of crescent microelectrode (a3), which avoids unprecedented motions at the sharp corners and minimizes the risk of biological damage due to high-intensity electric field.

Figure $2 b$ depicts the variation of square of electric potential along the vertical centerlines (black dashed lines in Fig. 2a) across the microelectrodes of different structures. The blue and red curves correspond to triangular and rectangular geometries, respectively, while the green curve corresponds to crescent geometry. Along all lines, the magnitude of electric potential decreases with the distance away from electrode edges, which obtains the maximum and minimum values at the electrode edge and center of the electrode structure, respectively. However, in the crescent microelectrodes, the electric field varies more evenly than 
Fig. 1 Simulation results showing the distribution of electric field (E, contours) and direction of negative DEP force (black arrows) in a vertical plane of microchannel patterned with electrodes on top and bottom surfaces. The height of the channel is $30 \mu \mathrm{m}$, and the applied voltage to the electrodes is $5 \mathrm{~V}$
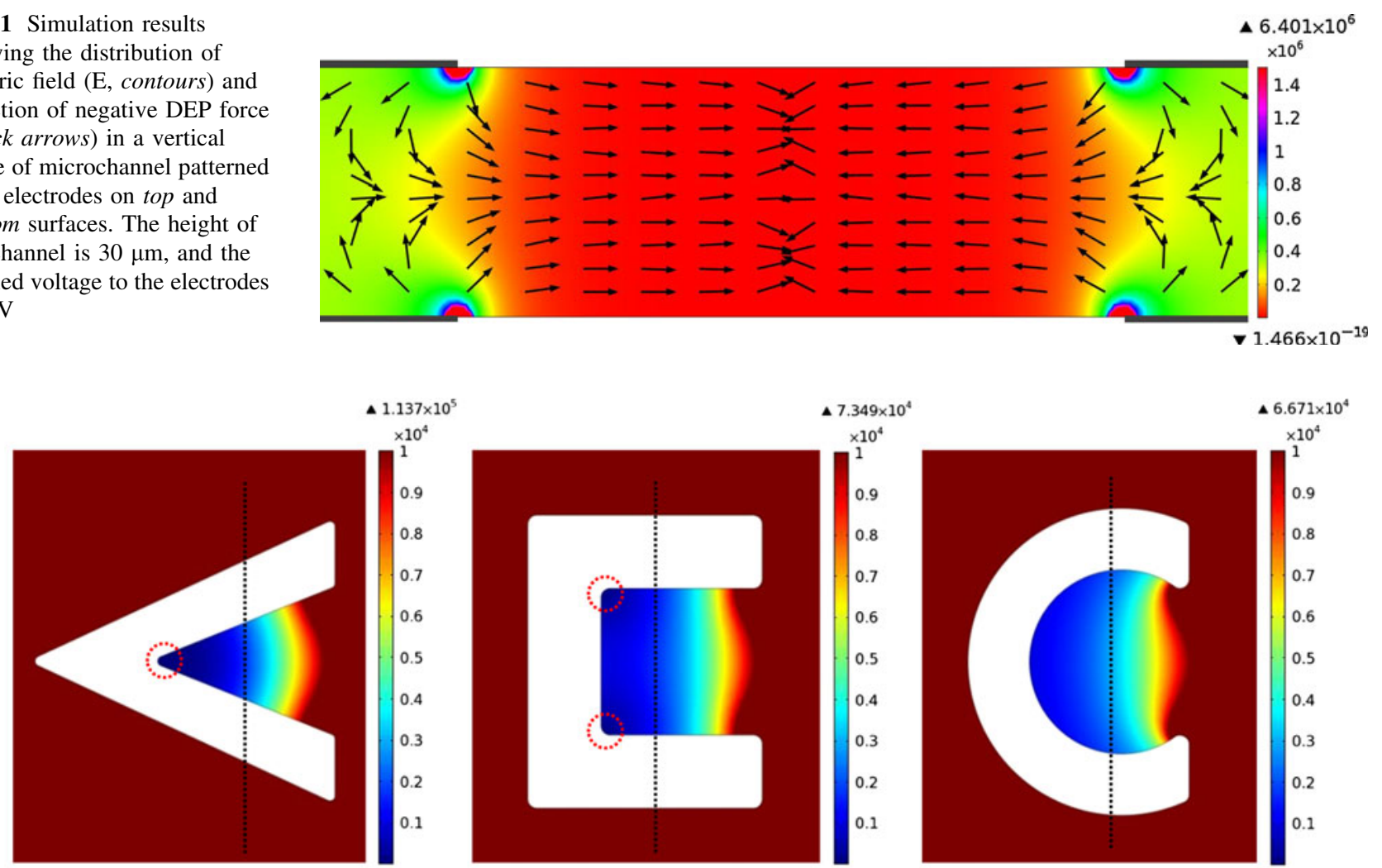

(a1)
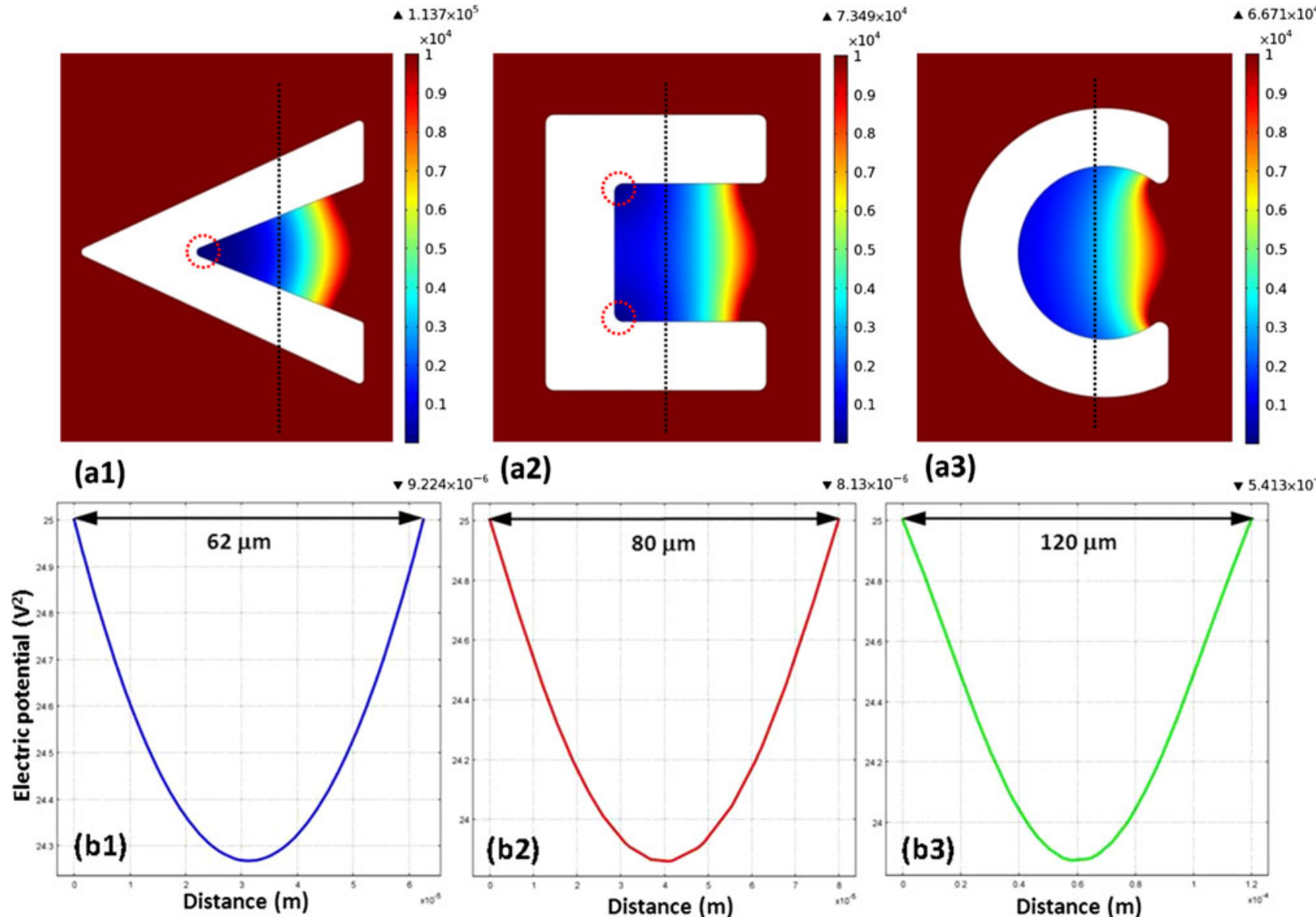

(a2)

(a3)
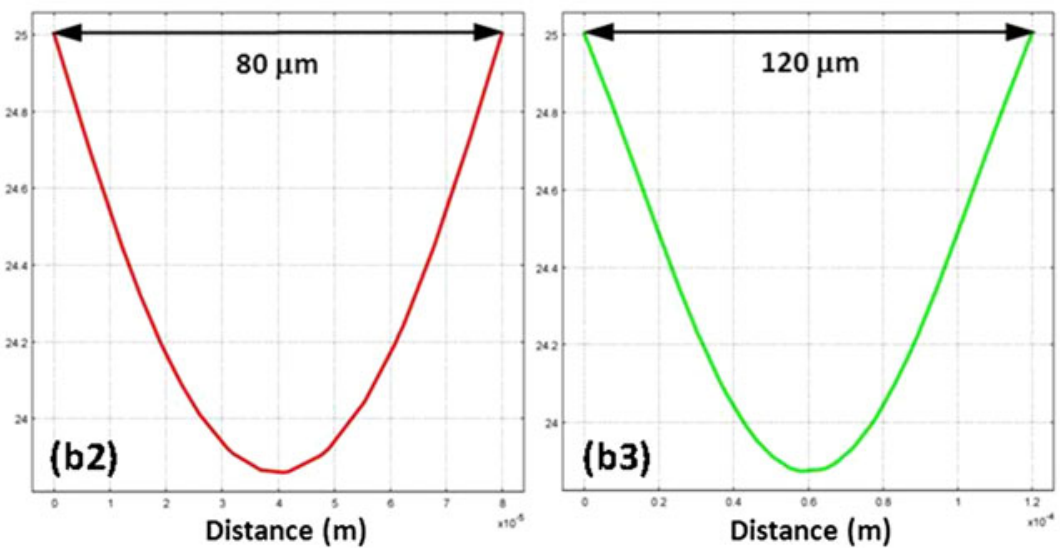

Fig. 2 Comparison of triangular (left column), rectangular (middle column), and crescent (right column) microelectrodes: a the distribution of electric field $(E)$ in the area formed by the geometry of microelectrodes; $\mathbf{b}$ the variation of the square of electric potential $\left(\varphi^{2}\right)$

other two types of electrode shapes (the potential magnitude decreases slowly as the distance from the electrode edge increased), and maintains over a longer distance $(120 \mu \mathrm{m})$, compared to triangular $(62 \mu \mathrm{m})$ and rectangular $(80 \mu \mathrm{m})$ ones. This suggests that crescent structure can increase the effective area inside the microelectrodes for DEP effect, therefore, more particles/cells can be accumulated within the microelectrode structure, producing a higher concentration yield. According to numerical results, crescent-shaped microelectrode shows such advantages as

along the vertical centerline (indicated by black-dash lines) across the microelectrodes. The applied field to the microelectrodes remained the same at $5 \mathrm{~V}$

the elimination of high-intensity electric field and the improvement of concentration yield.

\section{Experimental}

\subsection{Chip layout and fabrication}

A schematic representation of the dielectrophoretic chip with 3D microelectrode configuration for particle 


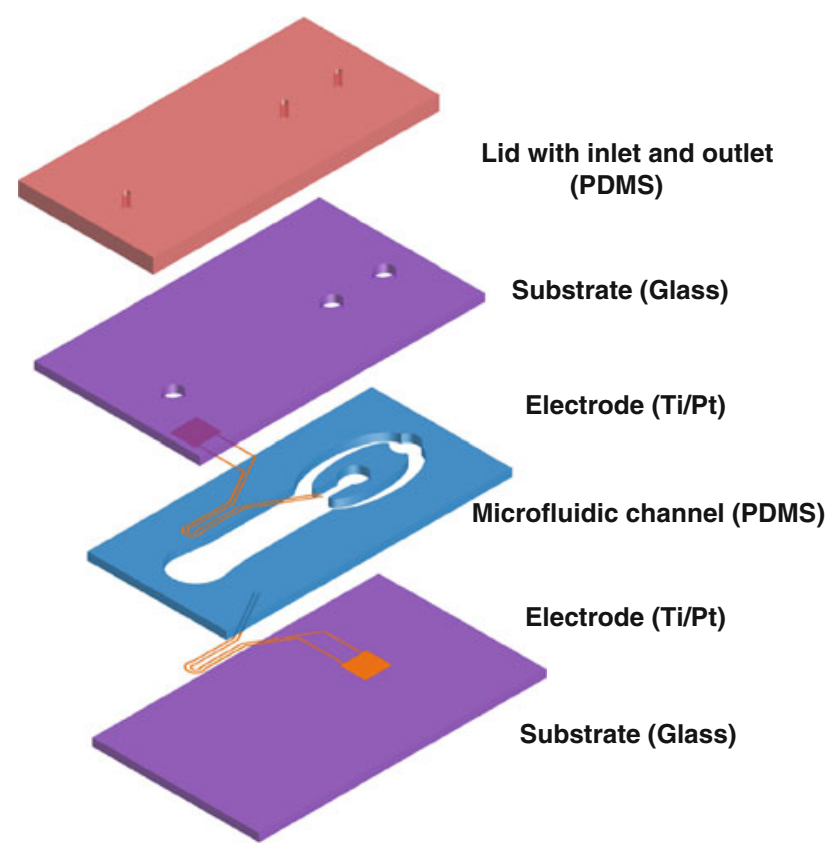

Fig. 3 Schematic diagram of the proposed micro-concentrator and separator using dielectrophoresis

manipulation is illustrated in Fig. 3, which is composed of two glass substrates with patterned microelectrode arrays, a polydimethylsiloxane (PDMS) layer containing microchannel sandwiched between the substrates, and a top PDMS lid with inlet and outlet for sample and buffer introduction. The proposed microchip was fabricated using a relatively simple and cost-effective method given elsewhere (Li et al. 2012). Microelectrode (20 nm Ti/100 nm $\mathrm{Pt}$ ) was made by standard photolithography and lift-off techniques, while microchannel with the depth of $30 \mu \mathrm{m}$ was created by patterning on a PDMS layer via laser ablation (VLS 2.50, Versa Laser System, Universal Laser System Ltd.). After constructing holes through the top glass substrate and PDMS lid, multiple layers (i.e. PDMS lid, PDMS-based microchannel, and microelectrodepatterned glass substrates) were bonded together using oxygen-plasma treatment (PDC-002, Harrick Plasma, NY, USA).

Figure $4 \mathrm{a}, \mathrm{b}$ presents the fabricated dielectrophoretic micro-concentrator/separator, and the plan view of the designed microelectrode array, respectively. The width of the electrodes and the gap between adjacent ones are both $40 \mu \mathrm{m}$, the length of the straight and oblique electrodes (placing at angle of $30^{\circ}$ to the flow direction) inside the PDMS-based microchannel are, respectively, 800 and $1,000 \mu \mathrm{m}$. The radius of the innermost crescent is $60 \mu \mathrm{m}$. The electric potential was applied through the pads with the dimension of $3 \times 3 \mathrm{~mm}$, placed on both top and bottom surfaces of the microchannel.

\subsection{Microelectrode configuration}

As indicated in Fig. 4b, the 3D electrode structure, where microelectrodes are patterned on both the top and bottom surfaces of the microchannel, was labeled into three units from right of left: focusing, aligning and trapping. The first microelectrode array takes advantage of funnel-shaped electrodes to focus target particles. Under the combination of dielectrophoretic and hydrodynamic force, target particles are driven laterally along the microelectrodes, and forced to the center of the microchannel as they passed through. Moreover, this electrode array can serve as a sorting unit for particle mixture, this is because target particles experiencing stronger DEP force are deflected and directed toward channel center region by the electrodes, while non-target particles pass over the electrodes and move along with the continuous flow toward the outlet due to hydrodynamic force.

The second array comprising parallel microelectrodes positioned symmetrically about the centerline of the channel serves to align target particles. The negative DEP forces from electrode arrays at both sides of the centerline can balance each other, focused target particles, therefore, are further directed and forced to the channel center region dielectrophoretically. As a result, target particles run with the flow in the direction parallel to the electrodes, and a confined and concentrated particle stream is formed along the channel centerline within the spacing between the inner straight microelectrodes. The upstream focusing and aligning units are essential, which are designed to line up the particles into a confined stream prior to entering the unit of trapping, resulting in improved trapping yield.

The final unit is used to trap and concentrate target particles after they were focused and aligned, which is composed of crescent-shaped microelectrodes created by intersecting concentric circles. Due to the negative DEP force, target particles are slowed down, retained, and finally prevented from passing through the microelectrodes. The intensity of trapped particles within the inner crescent-shaped electrodes increase over time, as the sample continuously flows through the microchannel. The captured target particles can be released and then collected at the outlet by turning off the applied electric field, making the proposed chip suitable for further detection and characterization of target particles.

\subsection{Apparatus}

The required sinusoidal voltages for particle manipulation were provided by a function generator (33120A, Agilent Technology, CA, USA), and a voltage amplifier (Stereo Power Amplifier 216THX). Two syringe pumps (PHD 2000, Harvard Apparatus, Holliston, MA) were employed 


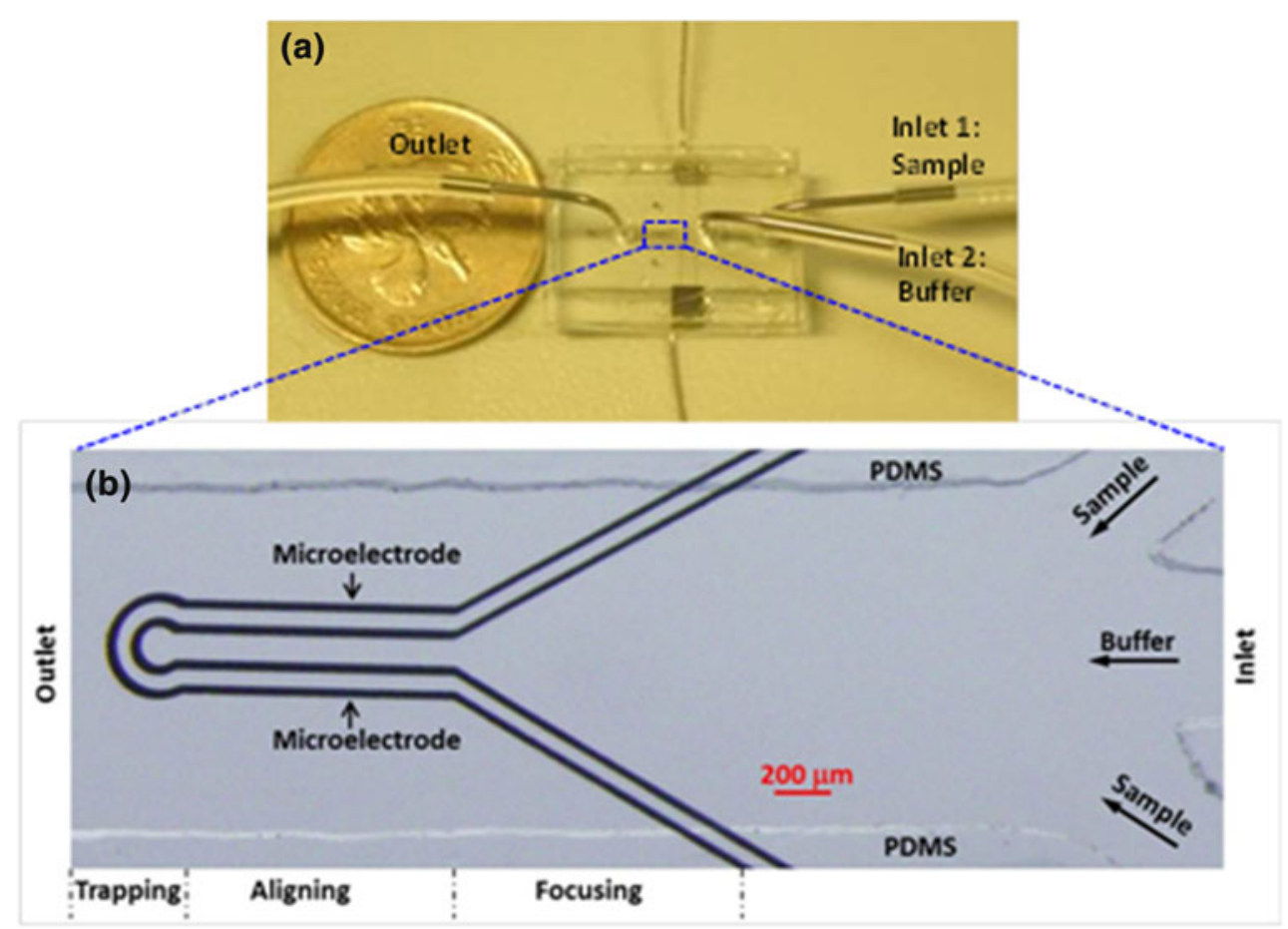

Fig. 4 a Photograph of the fabricated microfluidic chip used in the experiment for manipulating particles. b Magnified image indicating the structure of microelectrode array, which is composed of three units from right to left: focusing, aligning and trapping

to inject sample and buffer continuously into the microchannel. Two syringes were connected to the inlets through steel tube and capillary tubing (ID 1/32 inch, OD 3/32 inch Tygon tubing, Teflon ${ }^{\circledR}$ FER, Upchurch Scientific, USA). The motion of the particles and cells were observed and recorded using an inverted fluorescence microscope (IX71, Olympus, Tokyo, Japan) equipped with a CCD camera (DP 70, Olympus, Tokyo, Japan), and a computer with Olympus DP controller image software. Particle counting or concentration calculation was carried out using a Neubauer hemocytometer.

\subsection{Sample preparation}

The polystyrene particles with the diameter of 5 (SigmaAldrich, USA), 10 and $15 \mu \mathrm{m}$ (Fluosphere, Invitrogen, CA, USA) were selected for our experiments, as their sizes are comparable to biological cells such as yeast and red/white blood cells. Original 10 and $15 \mu \mathrm{m}$ particle suspensions were diluted by deionized (DI) water in the volume ratio of $1: 10$, while $5 \mu \mathrm{m}$ particles that originally suspended in pure water were re-suspended in $15 \mathrm{mM} \mathrm{NaCl}$ solution at a concentration around $10^{7}$ beads per milliliter. Baker's yeast cells (Saccharomyces cerevisiae) were cultured at $37{ }^{\circ} \mathrm{C}$ in the YEP broth (MP Biomedicals, LLC.). After about $24 \mathrm{~h}$, the cells were diluted with DI water three times and then re-suspended in $380 \mu \mathrm{S} / \mathrm{cm} \mathrm{NaCl}$ solution at a concentration of about $10^{7}$ cells per milliliter. For separation experiments, original $5 \mu \mathrm{m}$ particle solution was mixed with diluted 10 and $15 \mu \mathrm{m}$ particle solution, and yeast cell solution at a volume ratio of $1: 1,000$, respectively.

\section{Results and discussion}

\subsection{Concentration mechanism}

The presented microfluidic chip with 3D electrode configuration can be used to trap and concentrate particles and cells taking advantage of a force balance between negative DEP force and hydrodynamic force. With appropriate flow rate and sinusoidal voltage, target particles will be sequentially processed by the funnel-shaped, parallel and crescent-shaped microelectrodes, which are responsible for the effect of focusing, aligning and trapping, respectively. Figure 5 illustrates the overall dynamics of the target particles as they pass through the microchannel with a continuous flow. The net force resulting from the combination of dielectrophoretic and hydrodynamic force will determine the direction in which the particle will be moved. The movement of target particles in the four regions indicated by sequence numbers I, II, III and IV, from right to left are distinct: (I) Before approaching the electrode array, particles are driven to two streams close to the channel sidewalls due to the hydrodynamic force. (II) When particles reach the edge of the funnel-shaped electrodes, they are 


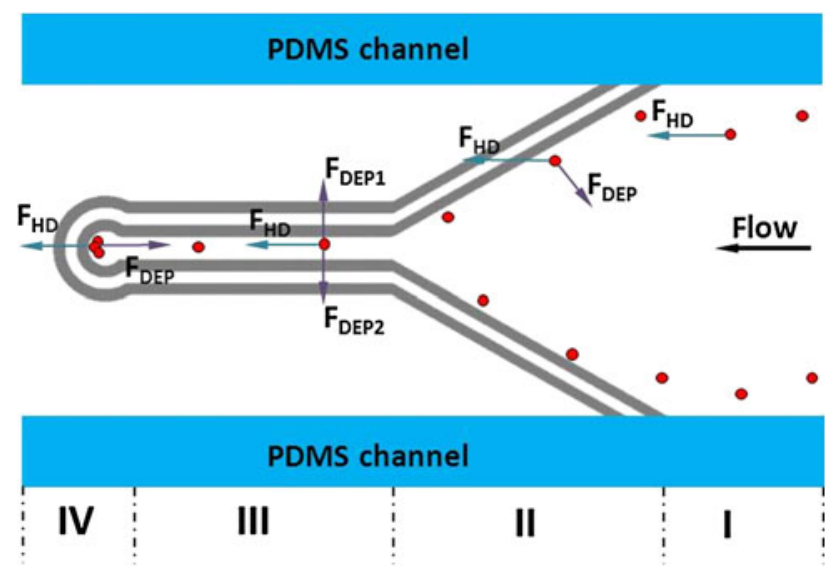

Fig. 5 Schematic dynamics of the concentration of target particles as they move in a continuous throughflow

deflected by negative DEP force and moved along the electrode length under the combined effect of two forces. Hence, they are gradually focused toward the region of channel center. (III) After travelling the length of oblique electrodes, particles continue through the channel within the spacing between inner parallel electrodes. Due to the negative DEP forces generated by the two sets of straight electrodes placed at both sides of the channel centerline, particles reach equilibrium positions where the DEP forces from these two sets of electrodes are equal, resulting in a confined particle stream along the centerline. (IV) The hydrodynamic force is overcome by the negative DEP force generated by the crescent-shaped microelectrodes, therefore, particles are slowed down, stopped and finally captured. With time, the trapped particles are accumulated into cluster, filling the region inside the inner crescentshaped electrodes.

\subsection{Concentration of polystyrene microparticles}

Figure 6 shows the overall movement of (a) 5, (b) 10 and (c) $15 \mu \mathrm{m}$ polystyrene particles at a flow rate of $0.3 \mu \mathrm{l} / \mathrm{min}$, and a field of $8 \mathrm{Vp}-\mathrm{p}$ and $10 \mathrm{kHz}$. At this frequency, all particles exhibited negative DEP effect and were focused, aligned, and trapped sequentially by the microelectrode array. As shown in the figure, particles carried by the continuous flow running from right to left were firstly deflected by funnel-shaped electrodes and moved along the oblique electrodes, continued to transport within the spacing between inner parallel electrodes, and finally trapped and accumulated at the area inside the inner crescent-shaped electrodes. The area of the concentrated particles at the trapping unit after applying electric field $40 \mathrm{~s}$ (right column) is larger than that after $10 \mathrm{~s}$ (left column), indicating a reliable performance of particle concentration with the continuous flow.
Consider a particle transport through the microfluidic channel, the DEP force generated by the microelectrode array competes against hydrodynamic force. Within a range of voltage, the DEP force is weaker than the hydrodynamic force, and the particle will be carried by the continuous flow toward the outlet. At a fixed flow rate, there exists a threshold voltage at which the particle begins to be deflected by the electrode array. The threshold voltage required for successfully trapping polystyrene particles of different sizes at varying flow rates (in the range between 0.3 and $0.8 \mu \mathrm{l} / \mathrm{min}$ ) was measured and plotted in Fig. 7. The inset shows the concentration of 5, 10 and $15 \mu \mathrm{m}$ particles at the trapping unit. The region inside the inner crescent microelectrode was seen full of high-density captured particles. The same experiment was conducted three times for each data point and the average values of the measurements were used. It can be found that the threshold voltage increased with the rise of flow rate, and was higher for smaller particles. According to Eqs. 1 and 4, DEP force and hydrodynamic force are proportional to the gradient of square of electric field and flow rate, respectively. Increased flow rate determines larger hydrodynamic force, therefore, larger electric field and correspondingly larger DEP force was required to counter increased hydrodynamic force for trapping fixed-size particles. Moreover, DEP force is proportional to the cube of particle radius, hence higher voltage is required for smaller particles to exhibit the same dielectrophoretic effect as larger particles.

\subsection{Concentration of yeast cells}

The performance of the proposed micro-concentrator was also examined by constantly trapping yeast cells while flowing in the microfluidic channel. The overall motion of yeast cells with an application of $12 \mathrm{Vp}-\mathrm{p}, 10 \mathrm{kHz}$ sinusoidal voltage and $0.2 \mu \mathrm{l} / \mathrm{min}$ flow rate is similar to that of polystyrene particles, as yeast cells experiencing strong negative DEP forces were sequentially focused, aligned and trapped by the electrode array. Figure 8 shows consecutive images of the trapping unit captured at intervals of $10 \mathrm{~s}$. The accumulation area of yeast cells increased over time, filling almost the region within the inner crescentshaped electrodes after $100 \mathrm{~s}$.

In addition, the cell-trapping efficiency was analyzed with respect to applied voltage and flow rate of sample. Cell counting of the yeast sample taken from outlet was carried out with a hemocytometer after the electric fields were applied. This method was also used to measure the cell concentration at the inlet. Hence, the trapping efficiency in each operation condition can be calculated by $\left(1-C_{\mathrm{o}} / C_{\mathrm{i}}\right) \times 100 \%\left(C_{\mathrm{o}}\right.$ and $C_{\mathrm{i}}$ are the concentration of yeast cells at the outlet and inlet, respectively). In Fig. 9a, the cell-trapping efficiency was plotted as a function of 
Fig. 6 Concentration of polystyrene particles of different sizes: a 5, b 10 and c $15 \mu \mathrm{m}$ at 10 (left column) and $40 \mathrm{~s}$ (right column). Target particles are sequentially focused, aligned and trapped by applying $8 \mathrm{Vp}-\mathrm{p}, 10 \mathrm{kHz}$ signal voltage and at a flow rate of $0.3 \mu \mathrm{l} / \mathrm{min}$
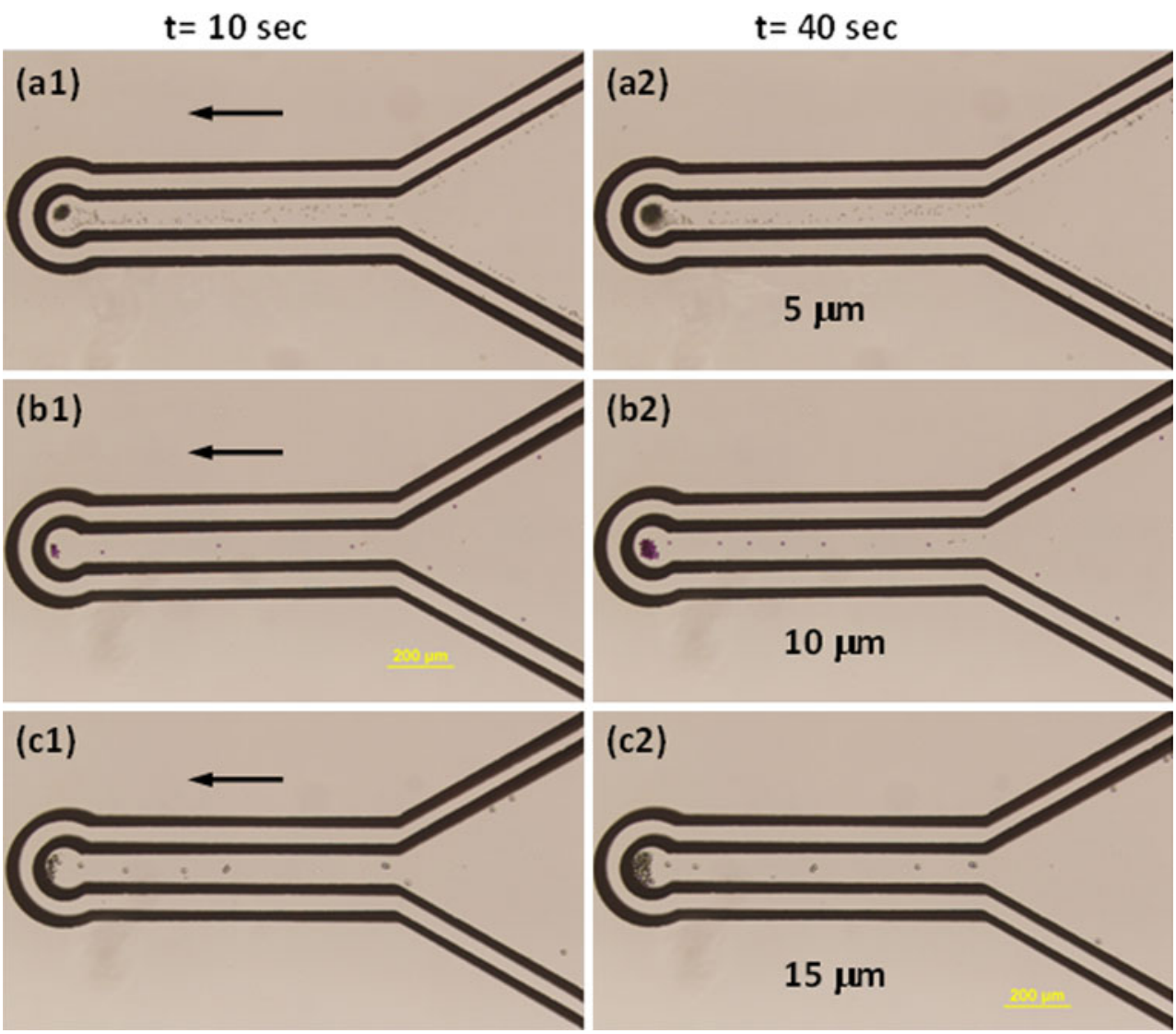

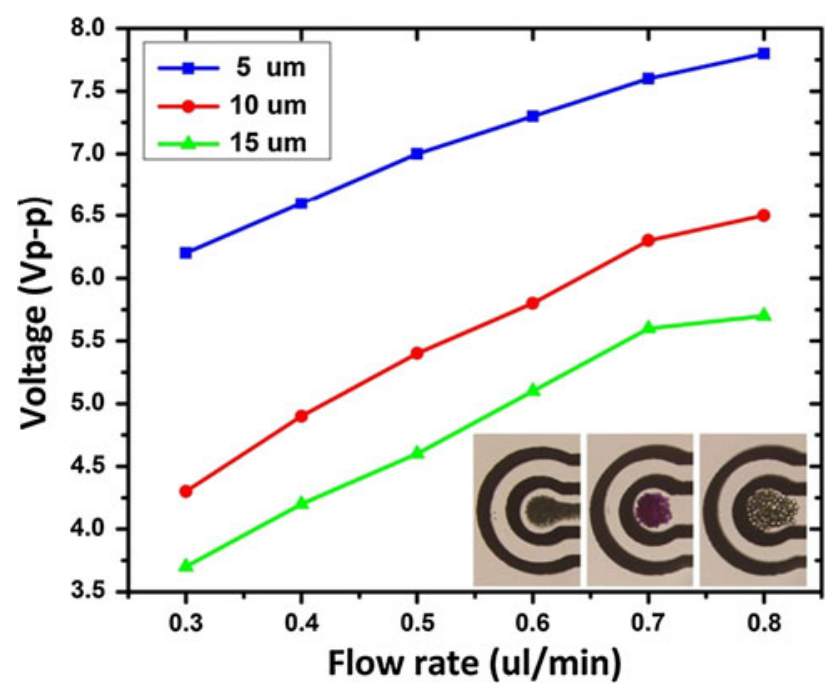

Fig. 7 Threshold voltages for trapping polystyrene particles with the diameter of 5,10 and $15 \mu \mathrm{m}$ at flow rate ranging from 0.3 to $0.8 \mu \mathrm{l} / \mathrm{min}$

applied voltage in the range between 7 and $12 \mathrm{Vp}$-p, with the flow rate and the field frequency kept constant at $0.3 \mu \mathrm{l} / \mathrm{min}$ and $10 \mathrm{kHz}$, respectively. It can be found that the trapping efficiency almost linearly depends on the applied voltage at a relatively low flow rate. This is mainly attributed to the fact that DEP force is proportional to the gradient of the square of electric field (see Eq. 1). An increased voltage leads to larger field gradient, and hence a larger DEP force for cell capture, resulting in improved trapping efficiency at a relatively low flow rate. Figure $9 \mathrm{~b}$ presents the variation of the cell-trapping efficiency with respect to the flow rate of cell sample for a fixed applied sinusoidal voltage of $9 \mathrm{Vp}-\mathrm{p}$ and $10 \mathrm{kHz}$. The trapping efficiency decreases with the rise of the flow rate. Given by Eq. 4, hydrodynamic force is linearly proportional to the flow rate. Increased flow rate determines correspondingly larger hydrodynamic force, which counteracts the effect of DEP force responsible for cell capture, and the trapping efficiency, therefore, decreases.

\subsection{Separation of polystyrene microparticles based on size}

Given by Eq. 1, DEP force is proportional to the cube of particle radius, thus larger particles will experience a stronger negative DEP force than smaller particles, and should be manipulated by microelectrode array more easily. The overall response of a mixture of particles having different sizes in the proposed micro-sorter is schematically illustrated in Fig. 10. The particle mixture was introduced into the microfluidic channel and hydrodynamically pre-confined into two streams near the channel sidewalls. 

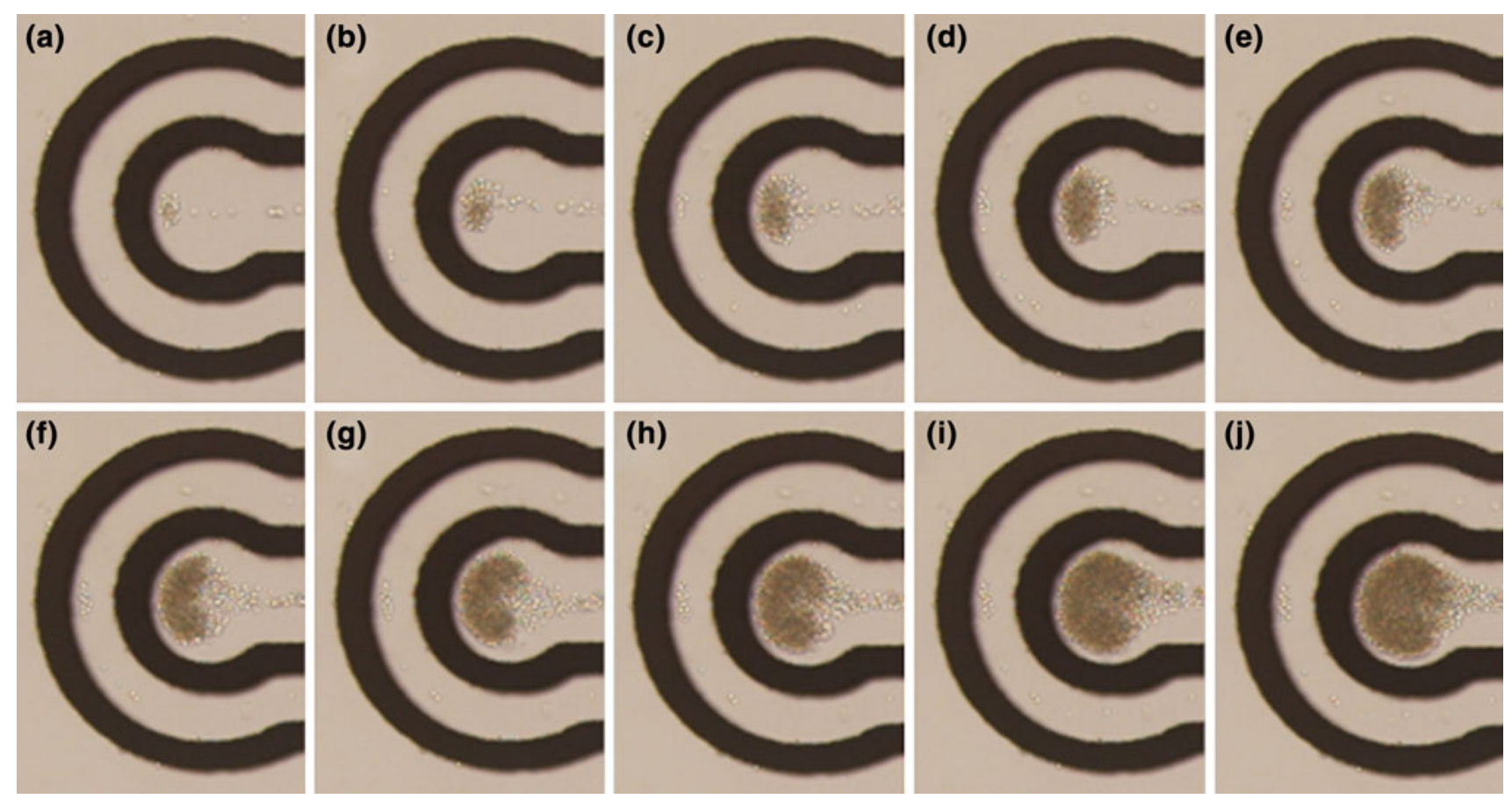

Fig. 8 Concentration of yeast cells at the trapping unit of crescent electrodes under a field of $12 \mathrm{Vp}-\mathrm{p}, 10 \mathrm{kHz}$ and a flow rate of $0.2 \mu \mathrm{l} / \mathrm{min}$ over $100 \mathrm{~s}$. Images were taken at $10 \mathrm{~s}$ intervals
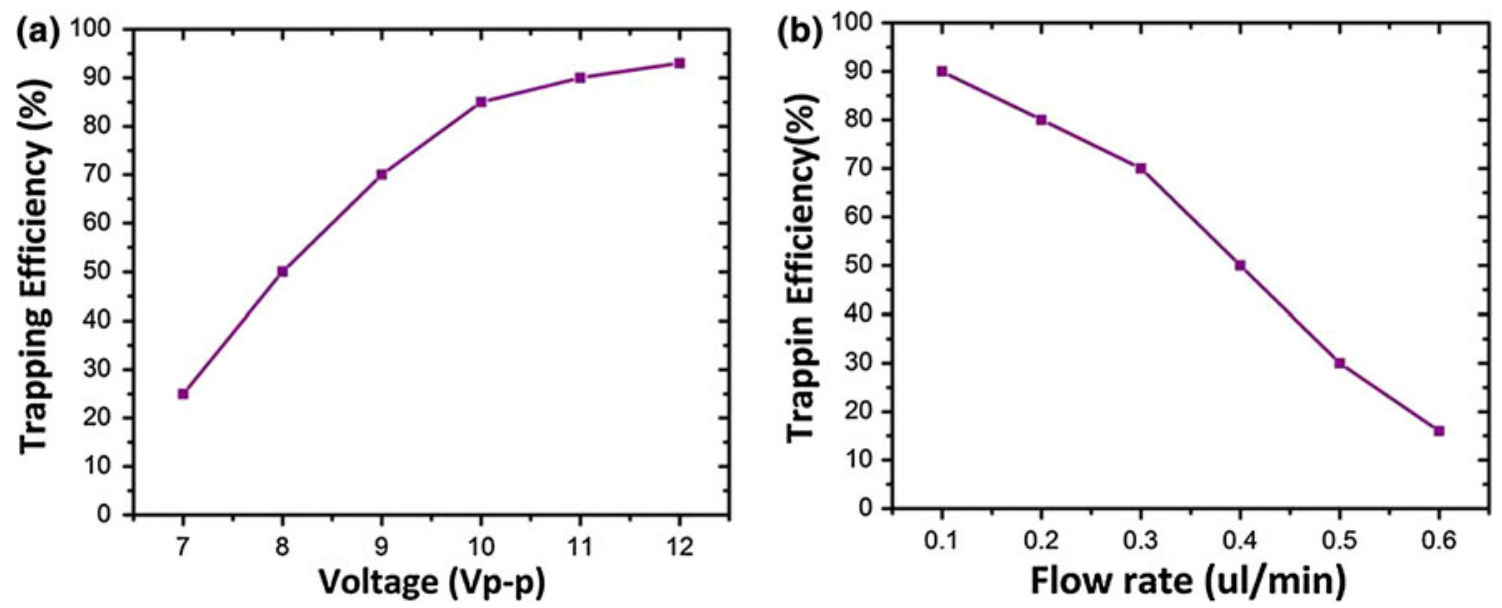

Fig. 9 a The effect of applied voltage on cell-trapping efficiency at a fixed flow rate and field frequency of $0.3 \mu 1 / \mathrm{min}$ and $10 \mathrm{kHz}$, respectively. b The effect of sample's flow rate on cell-trapping efficiency at a fixed signal field of $9 \mathrm{Vp}-\mathrm{p}$ and $10 \mathrm{kHz}$

As they move close to the microelectrode array, particles of different sizes were separated: large particles experiencing large negative DEP force will be deflected by oblique electrodes, and sequentially aligned and trapped by the straight and crescent microelectrodes, respectively; in contrast, small particles will pass over the funnel-shaped electrode array and flow to the outlet with the continuous throughflow. Figure 11 shows the separation of 5 and $10 \mu \mathrm{m}$ particles, when an AC signal of $6 \mathrm{Vp}-\mathrm{p}$ and $10 \mathrm{kHz}$ and a flow rate of $0.3 \mu \mathrm{l} / \mathrm{min}$ were provided. As expected,
$10 \mu \mathrm{m}$ particles were focused, aligned and trapped by the microelectrode array, while $5 \mu \mathrm{m}$ particles passed through the focusing unit of funnel-shaped microelectrodes with minor deflection. Similar results were obtained when the separation of a mixture of 5 and $15 \mu \mathrm{m}$ PS particles was examined at a flow rate of $0.3 \mu \mathrm{l} / \mathrm{min}$ with a $10 \mathrm{kHz}, 5 \mathrm{Vp}-\mathrm{p}$ sinusoidal voltage. As shown in the Fig. 12, the smaller particles $(5 \mu \mathrm{m})$ passed over the focusing unit and moved along with the flow, leaving the larger ones $(15 \mu \mathrm{m})$ manipulated by the electrode structure successfully. 


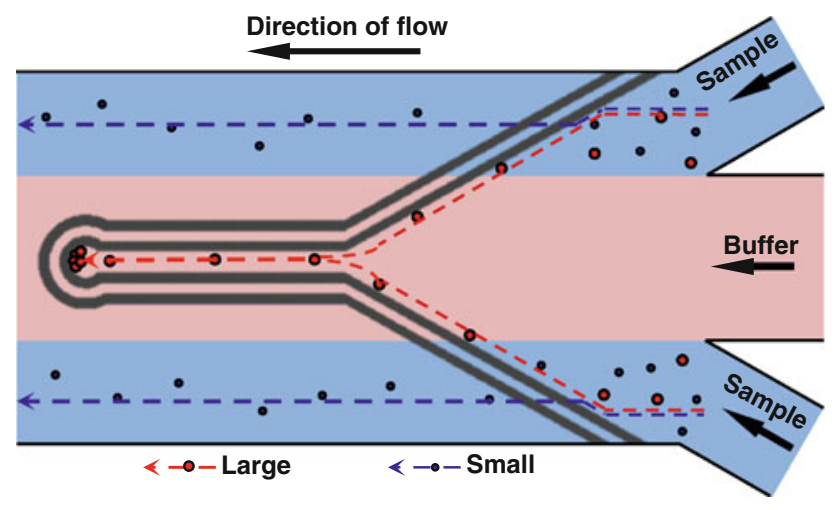

Fig. 10 Schematic dynamics of size-based particle separation. Large particles are focused, aligned and finally trapped by the microelectrode array, therefore, isolated from small particles carried away by continuous flow

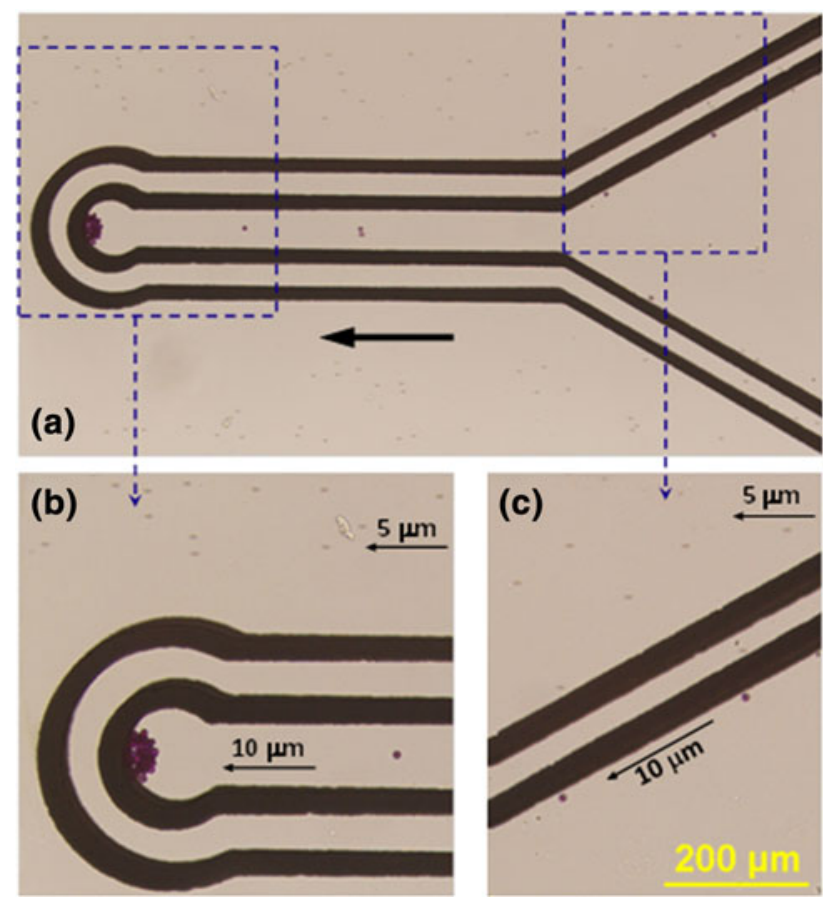

Fig. 11 Separation of 5 and $10 \mu \mathrm{m}$ particles at a flow rate of $0.3 \mu \mathrm{l} / \mathrm{min}$ with an applied sinusoidal voltage of $6 \mathrm{Vp}-\mathrm{p}$ and $10 \mathrm{kHz}$ : a overall response of the particle mixture at the microelectrode array, and magnified images showing the response of particles at the units of b trapping, and c focusing

\subsection{Separation of yeast cells and polystyrene microparticles based on dielectric properties}

Besides the separation depending on size, dielectric property-based separation was achieved by successfully separating $5 \mu \mathrm{m}$ diameter PS microspheres from yeast cells. The size of microspheres approximates to that of yeast cells, eliminating the effect of size on the separation

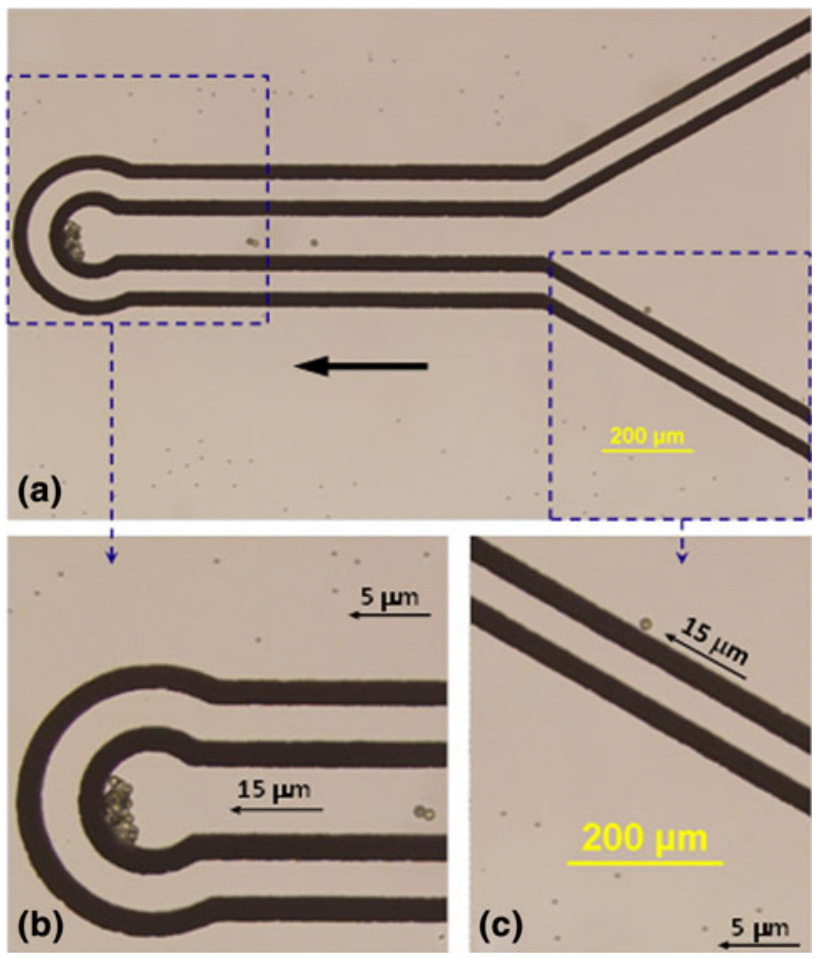

Fig. 12 Separation of 5 and $15 \mu \mathrm{m}$ particles at a flow rate of $0.3 \mu \mathrm{l} / \mathrm{min}$ with an applied sinusoidal voltage of $5 \mathrm{Vp}-\mathrm{p}$ and $10 \mathrm{kHz}$ : a overall response of the particle mixture at the microelectrode array, and magnified images showing the response of particles at the units of b trapping, and c focusing

performance. The sorting principle is that yeast cells and $5 \mu \mathrm{m}$ particles exhibit different DEP responses in a $380 \mu \mathrm{s} / \mathrm{cm} \mathrm{NaCl}$ solution at an AC signal of $300 \mathrm{kHz}$ (Lewpiriyawong et al. 2011): $5 \mu \mathrm{m}$ particles have a stronger negative DEP force due to large real part of CM factor, while yeast cells are near the cross-over frequency (real part of CM factor close to zero), which correspond to weak DEP force. Therefore, yeast cells can pass over funnel-shaped microelectrode with the continuous flow due to hydrodynamic force, while $5 \mu \mathrm{m}$ particles will be deflected by the electrode array, and finally concentrated at the trapping unit. Figure 13 illustrates the separation of yeast cells (white dots) and $5 \mu \mathrm{m}$ PS particles (black dots) at the trapping unit over $100 \mathrm{~s}$, under an applied $300 \mathrm{kHz}$, $7 \mathrm{Vp}$-p voltage and $0.2 \mu \mathrm{l} / \mathrm{min}$ flow rate. As polystyrene particles were being focused and aligned, they were trapped and concentrated in the inner crescent microelectrodes. The area of the concentrated particles increased as the time went by, preferring to fill the circular region at the trapping unit. $5 \mu \mathrm{m}$ PS particles, therefore, were sorted from yeast cells, which were seen moving to the outlet in two streams near both channel sidewalls. 
(a)

(b)

(c)

(d)

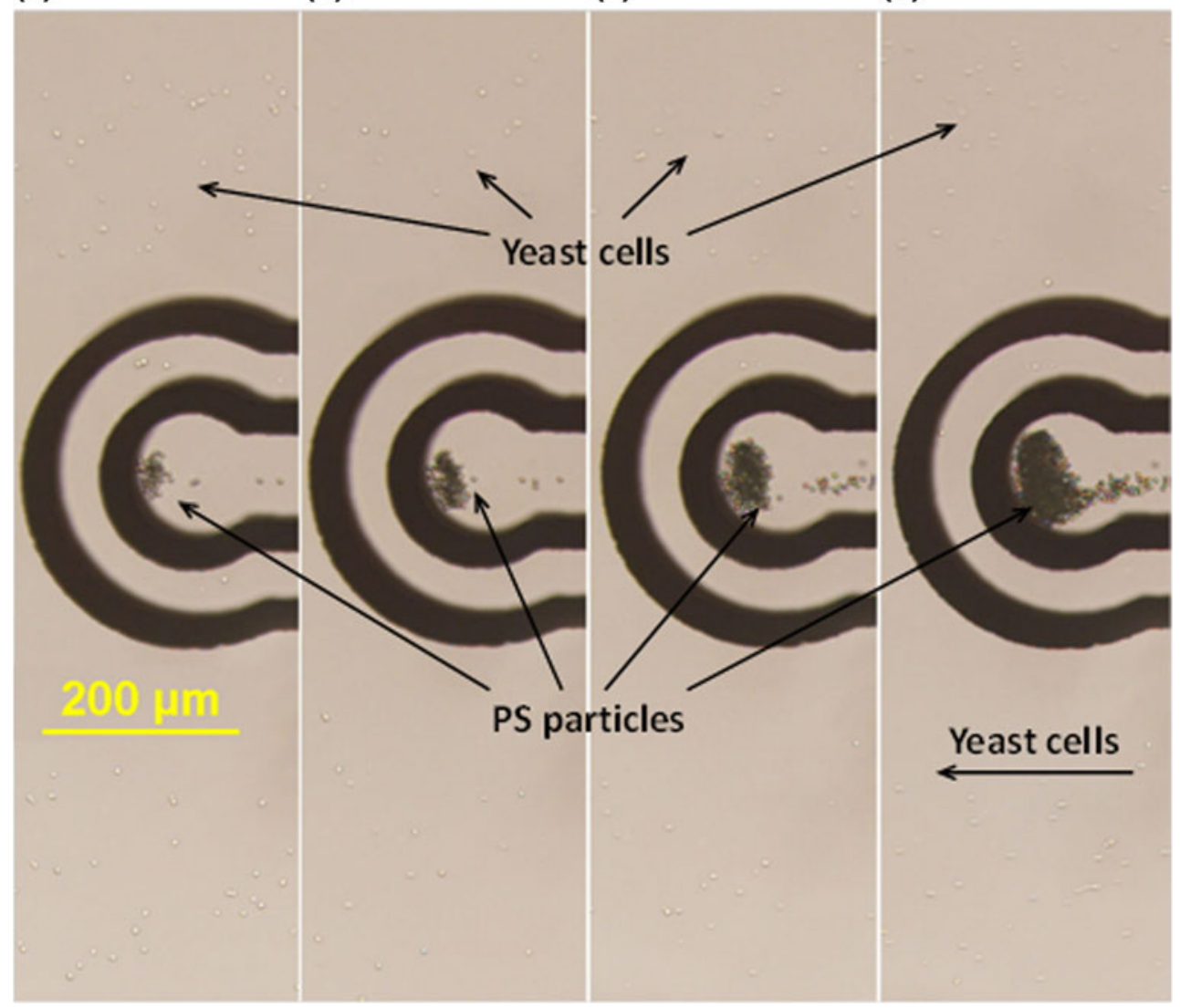

Fig. 13 Separation of yeast cells (white dots) and $5 \mu \mathrm{m}$ diameter polystyrene particles (black dots) at a flow rate of $0.2 \mu \mathrm{l} / \mathrm{min}$ with an applied sinusoidal voltage of $7 \mathrm{Vp}-\mathrm{p}$ and $300 \mathrm{kHz}$. The response of the mixture at the trapping unit over $100 \mathrm{~s}$ was shown: yeast cells

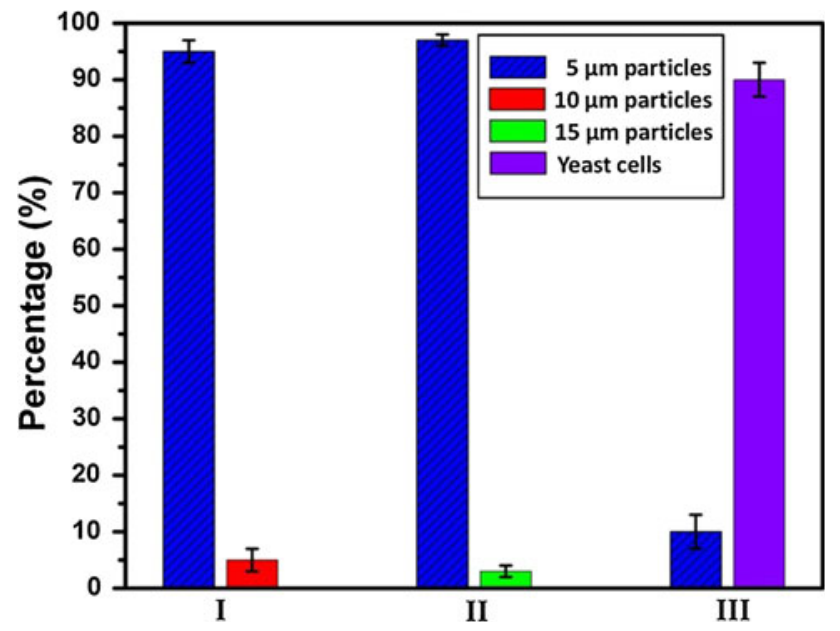

Fig. 14 Percentage of each particle population at the outlet in separation experiments: (I) size-based separation of 5 and $10 \mu \mathrm{m}$ PS particles, (II) size-based separation of 5 and $15 \mu \mathrm{m}$ PS particles, and (III) dielectric properties-based separation of yeast cells and $5 \mu \mathrm{m}$ PS particles were seen moving to the outlet with the continuous throughflow, while PS particles were successfully trapped within the crescentshaped electrodes, and the area of concentrated particles increased over time. a $t=10 \mathrm{~s}, \mathbf{b} t=25 \mathrm{~s}, \mathbf{c} t=50 \mathrm{~s}, \mathbf{d} t=100 \mathrm{~s}$

The performance of the proposed dielectrophoretic micro-separator was further evaluated in terms of relative percentage. After applying the electric field, the sample from the outlet was collected, and the number of each type of particle or cell was counted using a hemocytometer. The same procedure was repeated three times for each separation experiment, and both the average value and standard deviation were obtained. Figure 14 shows the percentage of: (I) 5 and $10 \mu \mathrm{m}$ particles, (II) 5 and $15 \mu \mathrm{m}$ particles, and (III) $5 \mu \mathrm{m}$ particles and yeast cells at the outlet, indicating high purity of particles which were not processed by the electrode array. Moreover, particle counting was conducted for the sample from the inlet, and the separation efficiency was calculated by $n_{\mathrm{r}} / n_{\mathrm{i}} \times 100 \%$, or $\left(1-n_{\mathrm{w}} / n_{\mathrm{i}}\right) \times 100 \%$, where $n_{\mathrm{i}}$ is the number of target particles at the inlet, $n_{\mathrm{r}}$ and $n_{\mathrm{w}}$ are number of target particles rightly and wrongly processed, respectively. The results showed that separation efficiency above $90 \%$ was achieved in all experiments. 


\section{Conclusions}

A DEP-based microfluidic device with 3D electrode configuration for continuous particle concentration and separation has been presented. For single-particle concentration, target particles running along with continuous flow are sequentially focused, aligned and trapped by the electrode array; for double-particle separation, particles that exhibit stronger negative DEP effect are deflected and manipulated, isolating from the other particle population moving toward outlet due to hydrodynamic force. The microdevice serving as both a microconcentrator and a microseparator was demonstrated using different-sized PS particles and yeast cells. The threshold voltage was obtained for PS particles with diameter of 5,10 and $15 \mu \mathrm{m}$, which depends on such factors as particle size and flow rate. Experimental results also indicated that cell-trapping efficiency can be improved by increasing signal voltage and decreasing operating flow rate in a certain range. Moreover, high efficiency above $90 \%$ was obtained in all separation experiments.

The proposed DEP-based microfluidic device has great advantages: (1) compared to planer electrodes only patterned on the bottom surface of the microchannel, 3D electrode configuration generates stronger DEP force over a larger volume of the microchannel for particle deflection, allowing shorter microchannel and higher operating flow rate, which leads to increased throughput; (2) the upstream units of focusing and aligning serve as pretreatment components, which direct target particles running with the flow toward the given trapping area, the capture efficiency hence improved; (3) large numbers of particles are trapped at a defined unique location rather than discrete locations along the microchannel, which facilitates post-processing, such as particle collection, detection and analysis; (4) versatile capabilities can be achieved, including single-particle concentration, and double-particle separation depending on size and dielectric properties; (5) it is easy in terms of fabrication and operation, because bonding top and bottom electrodes can be achieved by plasma treatment using a PDMS-based microchannel, and only single-channel AC power supply is required. The presented DEP-based microdevice is expected to be widely used for concentrating and separating particles of different sizes and dielectric properties in biological, medical and environmental fields.

\section{References}

Cetin B, Li D (2011) Dielectrophoresis in microfluidics technology. Electrophoresis 32:2410-2427

Cetin B, Kang Y, Wu Z, Li D (2009) Continuous particle separation by size via AC-dielectrophoresis using a lab-on-a-chip device with 3-D electrodes. Electrophoresis 30:766-772
Chen D, Du H (2007) A dielectrophoretic barrier-based microsystem for separation of microparticles. Microfluid Nanofluid 3:603-610

Cheng I-F, Chang H-C, Hou D, Chang H-C (2007) An integrated dielectrophoretic chip for continuous bioparticle filtering, focusing, trapping and detecting. Biomicrofluidics 1:021503

Dürr M, Kentsch J, Müller T, Schnelle T, Stelzle M (2003) Microdevices for manipulation and accumulation of micro-and nanoparticles by dielectrophoresis. Electrophoresis 24:722-731

Gossett DR, Weaver WM, Mach AJ, Hur SC, Tse HTK, Lee W, Amini H, Carlo DD (2010) Label-free cell separation and sorting in microfluidic systems. Anal Bioanal Chem 397:3249-3267

Iliescu C, Yu LM, Tay FEH, Chen BT (2008) Bidirectional field-flow particle separation method in a dielectrophoretic chip with $3 \mathrm{D}$ electrodes. Sens Actuators B 129(1):491-496

Iliescu C, Tresset G, Xu G (2009) Dielectrophoretic field-flow method for separating particle populations in a chip with asymmetric electrodes. Biomicrofluidics 3(4):044104

Jen C-P, Weng C-H, Huang C-T (2011) Three-dimensional focusing of particles using negative dielectrophoretic force in a microfluidic chip with insulating microstructures and dual planar microelectrodes. Electrophoresis 32:2428-2435

Jones TB (2003) Electromechanics of particles. Cambridge University Press, Cambridge

Kang Y, Li D (2009) Electrokinetic motion of particles and cells in microchannels. Microfluid Nanofluid 6:431-460

Khoshmanesh K, Nahavandi S, Baratchi S, Mitchell A, Kalantarzadeh K (2010) Dielectrophoretic platforms for bio-microfluidic systems. Biosens Bioelectron 26(5):1800-1814

Lewpiriyawong N, Yang C, Lam YC (2010) Continuous sorting and separation of microparticles by size using AC dielectrophoresis in a PDMS microfluidic device with 3-D conducting PDMS composite electrodes. Electrophoresis 31:2622-2631

Lewpiriyawong N, Kandaswamy K, Yang C, Ivanov V, Stocker R (2011) Microfluidic characterization and continuous separation of cells and particles using conducting poly(dimethyl siloxane) electrode induced alternating current-dielectrophoresis. Anal Chem 83:9579-9585

Li M, Li S, Wu J, Wen W, Li W, Alici G (2012) A simple and costeffective method for fabrication of integrated electronic-microfluidic devices using a laser-patterned PDMS layer. Microfluid Nanofluid 12:751-760

Pethig R (2010) Review Article-Dielectrophoresis: status of the theory, technology, and applications. Biomicrofluidics 4:022811

Pohl HA (1978) Dielectrophoresis: the behavior of neutral matter in nonuniform electric fields. Cambridge University Press, Cambridge

Pysher MD, Hayes MA (2007) Electrophoretic and dielectrophoretic field gradient technique for separating bioparticles. Anal Chem 79(12):4552-4557

Roda B, Zattoni A, Reschiglian P, MM H, Mirasoli M, Michelini E, Roda A (2009) Field-flow fractionation in bioanalysis: a review of recent trends. Anal Chim Acta 635(2):132-143

Schnell T, Müller T, Gradl G, Shirley SG, Fuhr G (1999) Paired microelectrode system: dielectrophoretic particle sorting and force calibration. J Electrostat 47:121-132

Song H, Mulukutla V, James CD, Bennett DJ (2008) Continuousmode dielectrophoretic gating for highly efficient separation of analytes in surface micromachined microfluidic devices. J Micromech Microeng 18:125013

Srivastava SK, Gencoglu A, Minerick AR (2011) DC insulator dielectrophoretic applications in microdevice technology: a review. Anal Bioanal Chem 399(1):301-321

Tai CH, Hsiung SK, Chen CY, Tsai ML, Lee GB (2007) Automatic microfluidic platform for cell separation and nucleus collection. Biomed Microdev 9(4):533-543 
Voldman J, Toner M, Gray ML, Schmidt MA (2003) Design and analysis of extruded quadrupolar dielectrophoretic traps. J Electrostatics 57(1):69-90

Wang L, Flanagan LA, Jeon NL, Monuki E, Lee AP (2007) Dielectrophoresis switching with vertical sidewall electrodes for microfluidic flow cytometry. Lab Chip 7(9):1114-1120

Wang L, Lu J, Marukenko SA, Monuki ES, Flanagan LA, Lee AP (2009) Dual frequency dielectrophoresis with interdigitated sidewall electrodes for microfluidic flow-through separation of beads and cells. Electrophoresis 30(5):782-791

Wlodkowic D, Cooper JM (2010) Microfabricated analytical systems for integrated cancer cytomics. Anal Bioanal Chem 398(1): 193-209
Xuan X, Zhu J, Church C (2010) Particle focusing in microfluidic devices. Microfluid Nanofluid 9:1-16

Yasukawa T, Suzuki M, Sekiya T, Shiku H, Matsue T (2007) Flow sandwich-type immunoassay in microfluidic devices based on negative dielectrophoresis. Biosens Bioelectron 22:2730-2736

Yu C, Vykoukal J, Vykoukal DM, Schwartz JA, Shi L, Gascoyne PRC (2005) A three-dimensional dielectrophoretic particle focusing channel for microcytometry applications. J Microelectromech Syst 14(3):480-487

Zhang C, Khoshmanesh K, Mitchell A, Kalantar-zadeh K (2010) Dielectrophoresis for the manipulation of micro/nano particles in microfluidic systems. Anal Bioanal Chem 396(1):401-420 